\title{
Hydrogen-Bond Interaction of PMAA/PVAc Blends: A Natural Abundant Two-Dimensional Exchange ${ }^{13}$ C NMR Investigation
}

\author{
Atsushi $\mathrm{ASANO}^{\dagger}$ \\ Department of Applied Chemistry, National Defense Academy, Yokosuka 239-8686, Japan
}

(Received July 15, 2003; Accepted October 29, 2003)

\begin{abstract}
The natural abundant two-dimensional exchange ${ }^{13} \mathrm{CNMR}$ revealed the very close proximity between the carboxyl carbon of PMAA and carbonyl carbon of PVAc in the miscible PMAA/PVAc $=3 / 1$ blend. The estimated distance between the CO carbons is approximately $0.37 \mathrm{~nm}$. This close distance is induced by a strong hydrogen bond between the hydrogen of carboxyl group for PMAA and the oxygen of carbonyl group for PVAc. The molecular mechanics (MM) calculation reveals that the distance of the hydrogen bond between the hydrogen of the carboxyl group and the oxygen of the carbonyl group is approximately $0.2 \mathrm{~nm}$ by fixing the intermolecular (inter-polymer) $\mathrm{C}-\mathrm{C}$ distance between the $\mathrm{CO}$ carbons to be $0.37 \mathrm{~nm}$. This value is reasonable for the very strong hydrogen bonding interaction. This interaction makes the ${ }^{13} \mathrm{C}$ chemical shift value of PVAc carbonyl carbon peak toward lower field by $4 \mathrm{ppm}$ and that of PMAA carboxyl carbon peak to change toward upper field by $4 \mathrm{ppm}$. The MM calculation also showed that the distance between the backbone carbons of PMAA and PVAc is less than $1 \mathrm{~nm}$. This very cross proximity for the interacted region is important to create the homogenous PMAA/PVAc blends on a scale of 2-5 nm.

KEY WORDS Hydrogen Bond / Polymer Blends / CPMAS ${ }^{13} \mathrm{C}$ NMR / Two-Dimensional Exchange ${ }^{13} \mathrm{CNMR} /$ Poly(methacrylic acid) / Poly(vinyl acetate) /
\end{abstract}

In the previous study, ${ }^{1}$ I showed that the excellent miscibility on a scale of $2-5 \mathrm{~nm}$ of the poly(methacrylic acid) [PMAA: $\left.-\left(\mathrm{CH}_{2} \mathrm{CCH}_{3}(\mathrm{COOH})\right)_{n}-\right]$ / poly(vinyl acetate) [PVAc: $-\left(\mathrm{CH}_{2} \mathrm{CH}\left(\mathrm{OCOCH}_{3}\right)\right)_{n}-$ ] blends is achieved by the specific intermolecular hydrogen bonding interaction at the unit molar ratio of $3: 2=[\mathrm{MAA}]:[\mathrm{VAc}]$ between the PMAA and PVAc: here, the word of "intermolecular" refers to "interpolymer" between PMAA and PVAc, while "intramolecular" means the inside of PMAA or PVAc, respectively. It has been also found that the amount of the intermolecular 3:2 = [MAA]:[VAc] interaction is needed over $30 \%$ to be a miscible blend on a scale of 2-5 nm. The PMAA-rich/PVAc blends have the interacted portion over $30 \%$, resulting that it shows the excellent miscibility on the $2-5 \mathrm{~nm}$ scale. On the other hand, the miscibility of the PMAA/PVAc-rich blends is not established on the $2-5 \mathrm{~nm}$ scale because the blends have the portion less than $25 \%$ only. ${ }^{1}$

The intermolecular hydrogen bonding is strong as the chemical shift of PVAc-CO carbon move toward the lower field by 4 ppm: the bulk PVAc-CO carbon resonate at $171 \mathrm{ppm}$ and the interacted $\mathrm{CO}$ carbon at $175 \mathrm{ppm}$. Furthermore, the interacted PMAA-COOH carbon is appeared at the upper field by $4 \mathrm{ppm}$ $(179 \mathrm{ppm})$ relative to the chemical shift value of the bulk PMAA-COOH carbon at $183 \mathrm{ppm}$; because the strength of intermolecular hydrogen bonding interaction is weaker than that of intramolecular PMAA, the peak of the intermolecular interacted PMAA-
$\mathrm{COOH}$ carbon shifts toward the upper field not but the lower field: the similar chemical shift change is observed in a PMAA/PEO complex ${ }^{3}$ and explained by the difference of the strength between the intermolecular and intramolecular hydrogen bonding. These shifts of the individual peaks produce the complicated ${ }^{13} \mathrm{C}$ NMR lineshape on the carboxyl/carbonyl (CO) region as shown in Figures 1 and 2 of reference 1.

The $3: 2=[\mathrm{MAA}]:[\mathrm{VAc}]$ unit ratio interaction causes a four-times contact situation to interact between the CO carbons comparing to the simple 1:1 interaction (the illustration is shown in Figure 1). In such a strong and specific interaction case, we will expect to be able to detect the natural abundant ${ }^{13} \mathrm{C}$ spin diffusion between the PMAA- $\mathrm{COOH}$ carbon at $179 \mathrm{ppm}$ and PVAc-CO carbon at $175 \mathrm{ppm}$. The ${ }^{13} \mathrm{C}$ spin diffusion cannot be detected over the distance of $1.0 \mathrm{~nm}$ because of the lower gyromagnetic ratio and the less natural abundant by comparison with those of ${ }^{1} \mathrm{H}$ nucleus. ${ }^{2}$ In this study, I show that the natural abundant two-dimensional (2D) exchange ${ }^{13} \mathrm{C}$ NMR experiment is one of useful tools to prove the existence of such an intermolecular specific interaction in the polymer materials.

\section{EXPERIMENTAL}

\section{Sample Preparation}

PMAA $\left(M_{\mathrm{w}}=150,000\right.$ and $\left.T_{\mathrm{g}}=491 \mathrm{~K}\right)$ obtained from Scientific Polymer Products, Inc. and PVAc

${ }^{\dagger}$ To whom correspondence should be addressed (E-mail: asanoa@nda.ac.jp, Fax: 046-844-5901). 


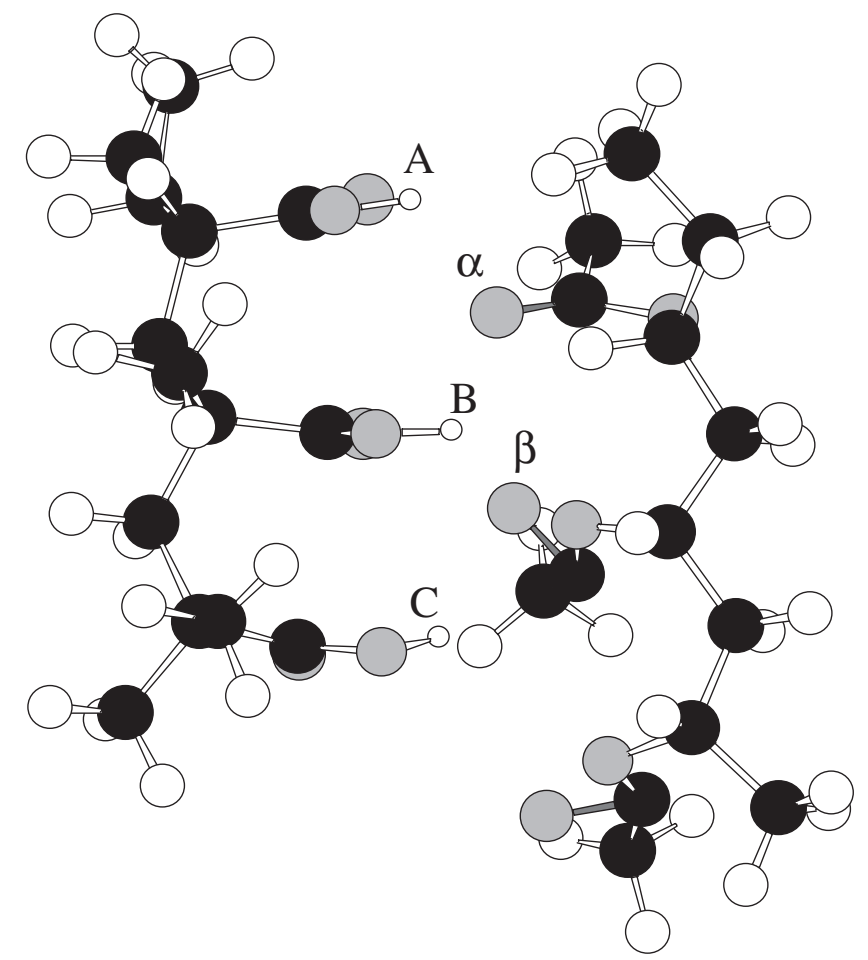

Figure 1. Illustration of the interacted portion for the PMAA/ PVAc blends. This figure is obtained from the molecular mechanical calculation by assuming that the distance of the carboxyl carbon of PMMA and the carbonyl carbon of PVAc is $0.37 \mathrm{~nm}$, and the interaction occurs at the molar unit ratio of $3: 2=$ [MAA]: [VAc]. Open circles represent hydrogens, black solid circles are carbons, and gray solid circles show oxygens. The symbols A, $\mathrm{B}$, and $\mathrm{C}$ represent hydrogens of carboxyl groups, and $\alpha$ and $\beta$ are carbonyl oxygens.

$\left(M_{\mathrm{w}}=167,000\right.$ and $\left.T_{\mathrm{g}}=292 \mathrm{~K}\right)$ obtained from Aldrich Chem. Co. Inc. were used without further purification. PMAA and PVAc were separately dissolved in methanol at a concentration of $10 \mathrm{w} / \mathrm{v} \%$ and mixed at desired molar unit ratios ([MAA]/[VAc]). The transparent films of PMAA/PVAc blends were obtained from casting the respective methanol solutions on a Teflon plate at $333 \mathrm{~K}$ and further dried under vacuum at $363 \mathrm{~K}$ for 4 days.

\section{NMR Measurements}

${ }^{13} \mathrm{C}$ NMR measurements were made using a Bruker DMX500 spectrometer operating at $125.76 \mathrm{MHz}$ for ${ }^{13} \mathrm{C}$ and $500.13 \mathrm{MHz}$ for ${ }^{1} \mathrm{H}$. High-resolution solidstate ${ }^{13} \mathrm{C}$ NMR spectra were obtained by the combined use of cross polarization (CP) and magic-angle spinning (MAS) with ${ }^{1} \mathrm{H}$ high-power dipolar decoupling (CPMAS). The radio-frequency field strengths for both ${ }^{13} \mathrm{C}$ and ${ }^{1} \mathrm{H}$ were $55.6 \mathrm{kHz}$ for Bruker probe and $50.0 \mathrm{kHz}$ for DOTY probe. The ${ }^{1} \mathrm{H}$ decoupling frequency was chosen to be $3 \mathrm{ppm}$ down-field from $\left(\mathrm{CH}_{3}\right)_{4} \mathrm{Si}$ (TMS) and the TPPM decoupling method ${ }^{4}$ is used. The MAS frequency is chosen to obtain a

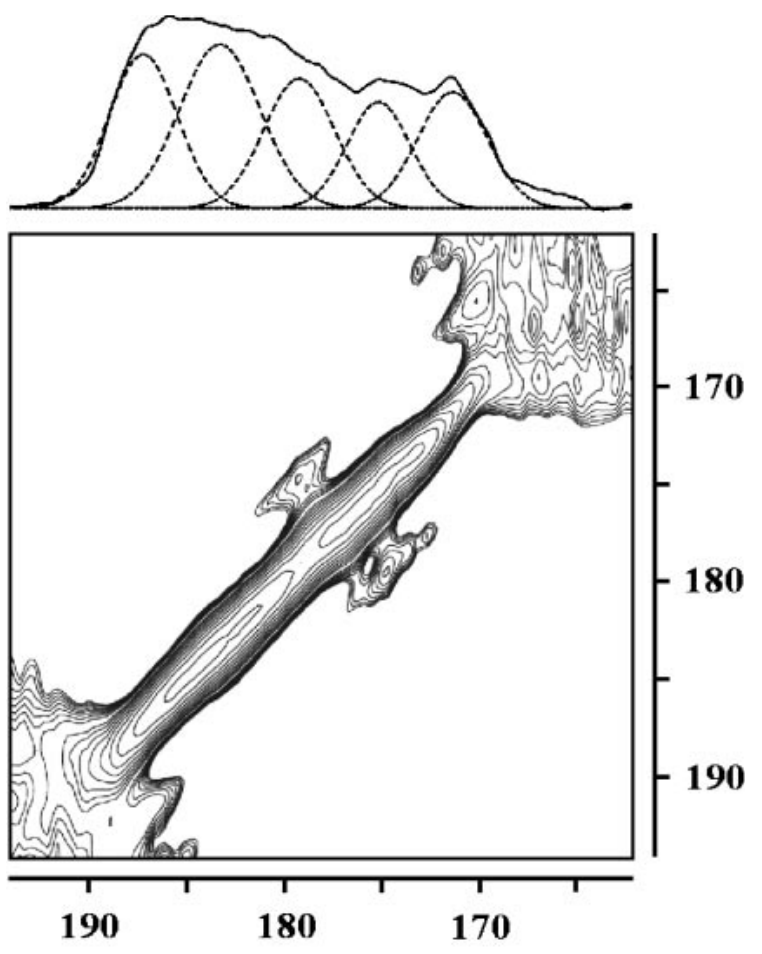

Chemical shift / ppm

Figure 2. Two dimensional exchange ${ }^{13} \mathrm{CNMR}$ spectrum in the $\mathrm{CO}$ region of the PMAA/PVAc $=3 / 1$ blend. The observed CPMAS ${ }^{13} \mathrm{CNMR}$ spectrum (a solid line) is shown on the top of the $2 \mathrm{D}$ spectrum with each decomposed simulated peak (a broken line). ${ }^{1}$ The cross peaks between $179 \mathrm{ppm}$ for $\mathrm{F}_{2}$ dimension and $175 \mathrm{ppm}$ for $\mathrm{F}_{1}$ dimension and between $175 \mathrm{ppm}$ for $\mathrm{F}_{2}$ dimension and $179 \mathrm{ppm}$ for $\mathrm{F}_{1}$ dimension are clearly observed. These cross peaks show the close proximity between those carbons. The mixing time of this figure is $7 \mathrm{~s}$. The $I_{\text {cross }} / I_{\text {diagonal }}$ intensity ratio is less than 0.2 and thus to show the small cross peak, the noise of foot of the broad diagonal peak is appeared in the corner of this figure.

clear spectrum at the aliphatic region without overlapping of the artificial spinning side bands of the $\mathrm{CO}$ carbons and set to $8 \mathrm{kHz} .{ }^{13} \mathrm{C}$ chemical shifts were measured relative to TMS using the methine carbon signal at $29.5 \mathrm{ppm}$ for solid adamantane as an external standard. The $2 \mathrm{D}$ exchange ${ }^{13} \mathrm{C}$ NMR spectrum were taken using time proportional phase increment mode with the TPPM ${ }^{1} \mathrm{H}$ decoupling and the ramped-amplitude ${ }^{5,6} \mathrm{CP}$. The $\mathrm{CP}$ contact time of $1.5 \mathrm{~ms}$ and mostly 280 scans with sampling points of 1024 for $\mathrm{F}_{2}$ dimension $\times$ sampling points of 128 for $F_{1}$ dimension are used. A series of the mixing times is $50 \mathrm{~ms}, 100 \mathrm{~ms}$, $150 \mathrm{~ms}, 200 \mathrm{~ms}, 250 \mathrm{~ms}, 300 \mathrm{~ms}, 500 \mathrm{~ms}, 1 \mathrm{~s}, 2 \mathrm{~s}, 3 \mathrm{~s}$, $4 \mathrm{~s}, 6 \mathrm{~s}$, and $7 \mathrm{~s}$. We examined the 2D exchange ${ }^{13} \mathrm{C}$ NMR measurement several times at each mixing time and used both Bruker and DOTY probes to check and distinguish the signal and noise. 


\section{RESULTS AND DISCUSSION}

The PMAA/PVAc blends have the specific $3: 2=$ [MAA]:[VAc] interaction, which is very strong as it produces $4 \mathrm{ppm}$ chemical shift change against both original chemical shifts of pure PMAA and pure PVAc. This characteristic unit ratio produces four contacts between a hydrogen of PVAc- $\mathrm{COOH}$ group and an oxygen of PVAc-CO group. The 3:2= [MAA]:[VAc] interacted portion is illustrated in Figure 1. Figure 1 is depicted by assuming that both chains have the isotactic and head-to-tail configurations. The symbols, $\mathrm{A}, \mathrm{B}$, and $\mathrm{C}$ represent the hydrogens of PMMA-COOH group and $\alpha$ and $\beta$ are the oxygen of PVAc-CO group. The hydrogen-bond contacts are achieved between $\mathrm{A}$ and $\alpha, \mathrm{B}$ and $\alpha, \mathrm{B}$ and $\beta$, $\mathrm{C}$ and $\beta$. We observe the three PMAA-COOH carbons at the same chemical shift value of $179 \mathrm{ppm}$, similarly the two PVAc-CO carbons at $175 \mathrm{ppm}$. If the carbonyl carbon of $\alpha$ is ${ }^{13} \mathrm{C}$, the probability of ${ }^{13} \mathrm{C}-{ }^{13} \mathrm{C}$ contact is twice (A and $\alpha$, B and $\alpha$ ) comparing to the simple 1:1 interaction. The same criterion is hold for the $\beta$ carbonyl carbon. This means that the ${ }^{13} \mathrm{C}-{ }^{13} \mathrm{C}$ exchange signal between the PMAA-COOH carbon and the PVAc-CO carbon is four-times enhanced in comparison with a general 1:1 interaction.

Figure 2 shows the $2 \mathrm{D}$ exchange ${ }^{13} \mathrm{CNMR}$ spectrum in the CO region of the PMAA/PVAc $=3 / 1$ blend. Since the peaks at 175 and $179 \mathrm{ppm}$ are more intense for the PMAA/PVAc $=3 / 1$ than the other blends, we chose the $3 / 1$ blend to detect the interpolymer cross peak. The spectrum clearly shows the cross peak between the peaks at 175 and $179 \mathrm{ppm}$. Because the single glass-transition of the miscible PMAA/PVAc $=3 / 1$ blend is found at around $440 \mathrm{~K}$, the molecular motion of those polymers is extremely slow at room temperature. Therefore, the effect of the molecular motion can be ignored, and then the cross peaks imply the existence of the intermolecular ${ }^{13} \mathrm{C}$ spin diffusion. These cross peaks indicate that the $\mathrm{COOH}$ carbon of PMAA observed at $179 \mathrm{ppm}$ is in very close proximity to the $\mathrm{CO}$ carbon of PVAc at $175 \mathrm{ppm}$ on a distance scale of less than $1.0 \mathrm{~nm}$, suggesting that both groups are strongly interacting with each other.

The cross/diagonal peak intensity ratio, $I_{\text {cross }} /$ $I_{\text {diagonal }}$ depends on the numbers of the interacted sites and those strengths, which can be interpreted by the proximity and/or the correlation time among the interacted sites. For the exchange experiment of the two-sites interaction, the growth rate of the $I_{\text {cross }} /$ $I_{\text {diagonal }}$ against the mixing time $\tau,\left[I_{\text {cross }} / I_{\text {diagonal }}\right](\tau)$ can be expressed by the following equation: ${ }^{7}$

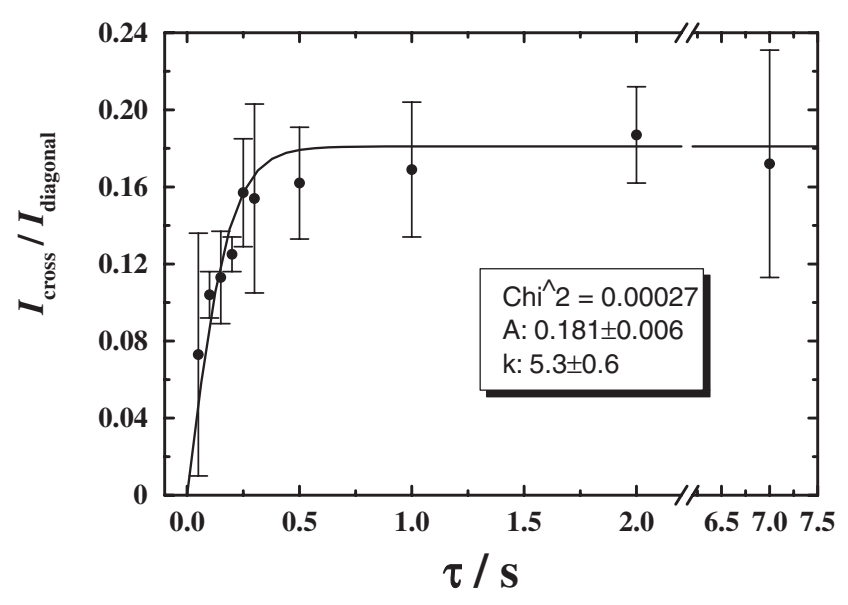

Figure 3. The $I_{\text {cross }} / I_{\text {diagonal }}$ intensity ratios against the mixing time. We measured several times for the same mixing time using both Bruker and DOTY probes. The $I_{\text {cross }} / I_{\text {diagonal }}$ ratios are averaged one for the two cross peaks and the values obtained from both probes. The solid curve is the "best-fit" line obtained from the least-square fit to eq 1 . The obtained ${ }^{13} \mathrm{C}$ spin-diffusion rate, $k$ is $5.3 \pm 0.6 \mathrm{~s}^{-1}$.

$$
\begin{aligned}
& {\left[I_{\text {cross }} / I_{\text {diagonal }}\right](\tau)=} \\
& \quad A[1+\exp (-2 k \tau)] /[1-\exp (-2 k \tau)] .
\end{aligned}
$$

Here, $A$ is the maximum cross peak intensity at $\tau=\infty$ and the parameter $k$ represents the ${ }^{13} \mathrm{C}$ spin-diffusion rate. The ${ }^{13} \mathrm{C}$ spin-diffusion rate depends on the minus 6-th power of the ${ }^{13} \mathrm{C}-{ }^{13} \mathrm{C}$ distance and the correlation time function of molecular motion associated with the ${ }^{13} \mathrm{C}-{ }^{13} \mathrm{C}$ dipolar interaction. ${ }^{2,7}$

Figure 3 shows the $I_{\text {cross }} / I_{\text {diagonal }}$ against the mixing time. The solid circles are the observed $I_{\text {cross }} / I_{\text {diagonal }}$ data points. Two cross peaks are obtained from the $2 \mathrm{D}$ exchange ${ }^{13} \mathrm{C}$ NMR experiment. The horizontal cross sections at $\omega_{1}=175$ and $179 \mathrm{ppm}$ give the $1 \mathrm{D}$ projections with the diagonal and cross peaks at the $\mathrm{CO}$ region. The obtained $I_{\text {cross }} / I_{\text {diagonal }}$ values corresponding to ${ }^{13} \mathrm{C}$ spin diffusion from PMAA-COOH group to PVAc-CO group (the cross peak at $\omega_{1}=$ $179 \mathrm{ppm}$ and $\omega_{2}=175 \mathrm{ppm}$ ) were sufficiently equaled to that from PVAc-CO group to PMAA-COOH group (at $\omega_{1}=175 \mathrm{ppm}$ and $\omega_{2}=179 \mathrm{ppm}$ ). The plotted $I_{\text {cross }} / I_{\text {diagonal }}$ ratios are averaged the values obtained from both cross peaks. The solid curve is the leastsquare fitted one using eq 1 . The relatively large deviations of the $I_{\text {cross }} / I_{\text {diagonal }}$ ratios are observed at the mixing times of $50 \mathrm{~ms}$ and $7 \mathrm{~s}$. At the shortest mixing time, it is difficult to detect the cross signal correctly because of a small peak intensity, and then I measured the $2 \mathrm{D}$ spectra much more times than the cases for longer mixing times and used both Bruker and DOTY probes. The size of each sample tube of these two probes is different: $4 \mathrm{~mm} \phi$ sample tube for Bruker probe and $5 \mathrm{~mm} \phi$ sample tube for DOTY probe. At first glance, it is preferable to use $5 \mathrm{~mm} \phi$ sample tube 
for getting the better signal to noise ratio, because of its greater sample volume. However, owing to our instrumental problem, the Bruker probe allows us to get the better stability of MAS and to use the higher power for getting a shorter $90^{\circ}$ pulse than the DOTY probe. These issues obscure the small cross peaks at the short mixing time. At a longer mixing time, a magnetic stability during a very long accumulation period becomes of great problem. The period of each $2 \mathrm{D}$ experiment was set to be less than one week to avoid the effect of the magnetic field drift. After finishing an accumulation, the magnetic field drift is calibrating again, and then the next accumulation was repeated and added to the previous accumulation to get the better signal to noise ratio. However, the large deviation for the long mixing time occurs even though such an effort is made.

The solid curve in the Figure 3 is the "best-fit" line obtained from the least-square fitting to eq 1 with the parameters of $A=0.181 \pm 0.006$ and $k=5.3 \pm$ $0.6 \mathrm{~s}^{-1}$. The fitting is in good agreement with the observed data points. The parameter $A$ seems very small. In this spin diffusion experiment case, the cross-peak intensity largely depends on the natural abundant of ${ }^{13} \mathrm{C}$ nucleus. For a ${ }^{13} \mathrm{C}$ in one of a pair, the probability is only $1.1 \%$ of having a ${ }^{13} \mathrm{C}$ in its partner site. ${ }^{8}$ In the early study of natural abundant 2D exchange ${ }^{13} \mathrm{C} \mathrm{NMR},{ }^{8,9}$ it is also showed that the observed cross peak intensity is about $10 \%$ of the diagonal peak.

It is noted that the $3: 2=[\mathrm{MAA}]$ :[VAc] relation produces the four-times enhancement for the $\left[I_{\text {cross }} / I_{\text {diagonal }}\right](\tau)$. Thus every $I_{\text {cross }}$ intensity is enhanced by four-times, resulting that both $A$ and $k$ become four-times greater values compared to a single ${ }^{13} \mathrm{C}-{ }^{13} \mathrm{C}$ pair contact. This indicates that we have to divide the $k$ value by 4 in order to obtain information on the intermolecular distance.

When we get a reference value for a parameter of $k$ with a known length, the distance between PMAA$\mathrm{COOH}$ and PVAc-CO carbons will be estimated. The best way to estimate the distance is to use an intramolecular cross peak intensity for PMAA or PVAc in the PMAA/PVAc blends and to obtain its $k$ value, for example, between the $\mathrm{C}(\mathrm{q})$ and $\mathrm{COOH}$ carbons for PMAA. However, even the $A$ value in the $3: 2=$ [MAA]:[VAc] interacted portion is only 0.18 . This means that the natural abundant single ${ }^{13} \mathrm{C}-{ }^{13} \mathrm{C}$ pair contact produces only the maximum 0.045 intensity against the diagonal peak. We cannot distinguish such a very small peak from the large noise and then it is not observable. Another way to get the correct $k$ value for a single ${ }^{13} \mathrm{C}-{ }^{13} \mathrm{C}$ pair contact in PMAA or PVAc polymers is to prepare the doubly ${ }^{13} \mathrm{C}$-enriched sample. This method however spends a lot of time and money to synthesize such a special sample, and has a problem whether the linear (no-branched) and the same molecular weight polymer can be synthesized. Furthermore, the enriched monomer produces not only the doubly enriched one but also multi-enriched one. The multi-enriched sample makes the analysis of the ${ }^{13} \mathrm{C}$ spin diffusion complicated.

The last (or easiest) way is to use the reported value for the other doubly ${ }^{13} \mathrm{C}$-enriched glassy polymer. The doubly $\mathrm{CH}_{2}{ }^{13} \mathrm{C}$-enriched poly(ethylene terephthalate) (PET) has been examined and the $k$ value of $19.0 \mathrm{~s}^{-1}$ is reported for the ${ }^{13} \mathrm{C}-{ }^{13} \mathrm{C}$ distance of $0.235 \mathrm{~nm}$ at room temperature. ${ }^{2}$ Since the value of $T_{\mathrm{g}}$ for PET is approximately $355 \mathrm{~K}$, the molecular motion is also negligible. Consequently, we estimate the intermolecular distance between PMAA and PVAc at the 3:2= [MAA]:[VAc] interacted portion by comparing the $k$ values between PET and the current polymer blends. The following eq 2 shows the relation between the $k$ values and the distances: $x$ indicates the distance between PMAA-COOH and PVAc-CO carbons.

$$
\frac{(5.3 \pm 0.6) / 4}{19.0}=\frac{0.235^{6}}{x^{6}}
$$

From the eq 2, the distance between PMAA-COOH and PVAc-CO carbons is obtained as $0.37 \pm 0.04 \mathrm{~nm}$.

The shortness of the obtained value of $0.37 \mathrm{~nm}$ is caused by the strong hydrogen bond between PMAA-COOH group and PVAc-CO group. To estimate the distance of the hydrogen bond, I simulated the minimum conformational energy state for the $3: 2=[\mathrm{MAA}]:[\mathrm{VAc}]$ interacted portion by fixing the distance between the $\mathrm{CO}$ carbons to be $0.37 \mathrm{~nm}$ by means of the molecular mechanical (MM) calculation. To facilitate the calculation, we used the respective three-unit chains, that is, $\mathrm{CH}_{3}$-MAA-MAA-MAA$\mathrm{CH}_{3}$ and $\mathrm{CH}_{3}-\mathrm{VAc}-\mathrm{VAc}-\mathrm{VAc}-\mathrm{CH}_{3}$ as shown in Figure 1.

The obtained hydrogen-bond distances between $\mathrm{A}$ and $\alpha, \mathrm{B}$ and $\alpha, \mathrm{B}$ and $\beta, \mathrm{C}$ and $\beta$ are approximately $0.2 \mathrm{~nm}$. This distance is comparable to the hydrogen bond length for a low-molecular-weight carboxylic acid dimers (ca. $0.18 \mathrm{~nm}$ ). This shortness proves the large chemical shift change of $4 \mathrm{ppm}$ for the blends as observed in the ${ }^{13} \mathrm{C}$ NMR spectra. ${ }^{1}$ The extent of the chemical shift change depends on the strength of the hydrogen bond and the strength is related to the distance. The shorter the distance becomes the larger the chemical shift changes towards lower field. In this current study, the $3: 2=[\mathrm{MAA}]:[\mathrm{VAc}]$ relation is formed by two doubly $\mathrm{COOH}-\mathrm{CO}$ contacts (Figure 1 ; e.g., a doubly contact refers to $\mathrm{A}$ and $\alpha$, $\mathrm{B}$ and $\alpha$.). This relation, hence, produces twice shifts: the net shift per a $\mathrm{COOH}-\mathrm{CO}$ contact roughly becomes half, 2 ppm.

Furthermore, the MM calculation shows that the 
distance between the backbone chains is less than $1 \mathrm{~nm}$ at the interacted part. The previous study ${ }^{1}$ revealed that the amounts of the interacted portion are needed over $30 \%$ to be miscible on a scale of a few $\mathrm{nm}$. Therefore, it is not necessary for all chains to be in close proximity with each other but if a few $10 \%$ of chains are in very close proximity by a specific interaction the other chains cannot separate more than $10 \mathrm{~nm}$, resulting the molecular-level homogeneity on a scale of a few $\mathrm{nm}$ is achieved in the whole blends.

\section{CONCLUSION}

I showed that the natural abundant 2D exchange ${ }^{13} \mathrm{C}$ NMR can be useful and powerful tool to detect and investigate an interacted portion in the case of a strong and specific interaction. Nevertheless, this experiment has an inconvenience that it takes long time. I spent several months on accomplishing the $2 \mathrm{D}$ exchange ${ }^{13} \mathrm{C}$ NMR experiments. However, this problem will be overcome by using a much higher magnetic field or a larger sample tube.

\section{REFERENCES}

1. A. Asano, M. Eguchi, M. Shimizu, and T. Kurotsu, Macromolecules, 35, 8819 (2002).

2. M. Linder, P. M. Henrichs, J. M. Hewitt, and D. J. Massa, J. Chem. Phys., 82, 1585 (1985).

3. T. Miyoshi, K. Takegoshi, and K. Hikichi, Polymer, 37, 11 (1996).

4. A. E. Bennet, C. M. Rienstra, M. Auger, K. V. Lakshmi, and R. G. Griffin, J. Chem. Phys., 103, 6951 (1995).

5. O. B. Peersen, X. Wu, I. Kustanovich, and S. O. Smith, J. Magn. Reson. A, 104, 334 (1993).

6. G. Metz, X. Wu, and S. O. Smith, J. Magn. Reson. A, 110, 219 (1994).

7. R. R. Ernst, G. Bodenhausen, and A. Wokaun, "Principles of Nuclear Magnetic Resonance in One and Two Dimensions", Oxford University Press, London, 1961, Chapter 9.

8. K. Schmidt-Rohr and H. W. Spiess, "Multidimensional Solid-State NMR and Polymers", Academic Press, San Diego, 1994, Chapter 13, pp. 426 \& 429.

9. N. M. Szeverenyi, M. J. Sullivan, and G. E. Maciel, J. Magn. Reson., 47, 462 (1982). 